\title{
A Micro-view on Techniques for Knowledge Based Systems
}

\author{
Ravi Lourdusamy, Xavierlal J. Mattam
}

\begin{abstract}
Knowledge representation has been a topic of research and study from the time machine processing of data and information became popular. The attempt to mimic the human cognition using artificial intelligence techniques has been the goal of artificial intelligence systems. The crux of cognition is knowledge. Knowledge is an inference made from data and information that is acquired from a variety of sources. The ability to efficiently acquire information, process it and store it as a knowledge base for further retrieval and use makes the artificially intelligent system a cognitive system. The paper is a brief introduction to knowledge representation techniques and schemes.
\end{abstract}

Keywords:--Artificial intelligence, Database, Knowledge base systems, Knowledge representation

\section{INTRODUCTION}

The successful implementation of any artificial intelligence system depends wholly on the efficiency and robustness of its knowledge representation techniques. Systems using artificial intelligence can be of two types. The more popular knowledge based systems and the less used non-knowledge based systems. In both the systems one or more artificial intelligence algorithms are used. The supervised and the unsupervised techniques in artificial intelligence depend on the dataset used for the machine learning. All the datasets have to be represented in the system and the effectiveness of the algorithm depends on the reliability of the datasets. The study of the knowledge representation techniques helps overcome challenges in using datasets especially in knowledge based systems.

In this paper an attempt is made to introduce the knowledge representation techniques that are popularly used in knowledge based system. Thesectionfollowing it is a description of knowledge based systems. After that, few popular knowledge representation techniques are discussed. Before the final discussion and conclusion, a comparison is made between relation database and graph databases that are the two currently common storage schemes of data storage.

\section{KNOWLEDGE BASED SYSTEMS (KBS):}

KBS are computer systems or programs that has a repository of knowledge known as the knowledge base which is used for making inferences through a computational process. The creation of the knowledge base requires the acquisition of information from structured and unstructured sources and the conversion of these data into

Revised Manuscript Received on December 30, 2019.

Dr. Ravi Lourdusamy, Associate Professor \& Head of the Department, Department of Computer Science, Sacred Heart College (Autonomous), Tirupattur, TN - 635 601, India.

Xavierlal J. Mattam, Research Scholar, Ph. D. Management (full time), Department of Computer Science, Sacred Heart College (Autonomous), Tirupattur, TN - 635 601, India. machine processable formats. There are three parts in a KBS; the knowledge base, the reasoner or the inference engine and the interface system. [1] KBS are also known as expert systems or intelligent system or the knowledge systems in general. The earliest type of KBS is the rule based systems. Later the model based systems evolved and currently the cognitive KBS with artificial intelligence, evolutionary knowledge development and multisource knowledge acquisition is developed. [2]Rule based KBS provides constant interaction between multiple rules derived from expert knowledge. The rules remain in a 'black box' and is not visible to the user. The two types of rule based systems are the backword chaining system or the logical programming systems and the forward chaining systems or the production systems. The rules in both the types are used for inferencing but are meant for problems of different types and use rules differently. The backword chaining methods usually use the 'if-then' condition to logically evaluate each element and move from one condition to another till a final condition is met. If at any stage, the condition is not fulfilled, the backtracking method is used to move to alternate conditions from the previous positive condition. In forward chaining system, the production rules are used to evaluate the data stored in the 'working storage'. If the rule is met by the data, then an action is performed using the data based conditions. If the rule is not met, a different rule is used from the set of production rules.[3]

The problem with rule based KBS is the need for a constant check for anomalies in the rules. The anomalies can arise either due to omission of certain rules that affect the inference which is classified as redundancy of rule or because there occurs wrong inferences due to the wrong data that is entered which is classified as conflict or when an inference is based on the same inference which is classified as circularity or no inference can be made from a set of input data which is classified as deficiency. [4]

In the model based KBS a model of the knowledge base is first formulated and then in the design and implementation phase the model is worked out to check if the model fits the required result. If there is any problem in obtaining the result, the model is modified or changed. The emphasis on creating the model allows the design and implementation to be effortless and smooth. The modelling consists of two parts. The first part of the model consists of the domain knowledge and the second part consists of the problem solving. Both the parts can be separate models for more autonomy and reusability. A crucial element of the model based system is the knowledge acquisition model.[5]. 


\section{A Micro-view on Techniques for Knowledge Based Systems}

Modelling techniques can help in the building modelling templates that will in turn contribute in either creating new models or in the reuse or extending the existing models without much effort. The portability and flexibility of the model is an added advantage while creating new knowledge based systems. [6] the constant updating of knowledge. The earlier KBS required a complete creation and testing of the knowledge base so anomalies creep in when new knowledge is acquired. Another problem with the rule based system was that it was too slow, fragile and limited. The Artificial Intelligence (AI) based systems or the intelligent system automatized the knowledge acquisition and validation process making the KBS robust, efficient, reliable and relevant. Therefore, the KBS began to be used in many domains and were commercialised. [7]

\section{KNOWLEDGE REPRESENTATION TECHNIQUES}

The language used for human communication is termed as the natural language. Natural language is human readable but it is not easily machine processable. The machine processable language is formal language. Knowledge representation is computational models to represent natural language in machine processable form in order to acquire or infer knowledge from structured and unstructured data sources. Knowledge representation is also the central problem in AI based KBS. The different ways of knowledge representation techniques can be broadly classified into three: Logical knowledge representation, Semantic knowledge representation and frame based knowledge representation. [8], [9]

\subsection{Logical Knowledge Representation [8-12]}

In logical knowledge representation, the natural language sentences are broken into propositions that can be written in logical relations. Constants, variables, functions, predicate, connectives and qualifiers are used to create formulas using any one of a variety of logic such as predicate, first order or higher order, multi-valued, lambda calculus, modal, fuzzy and so on. The knowledge base of the KBS with logical knowledge representation contains a collection of formulas that can be manipulated by the addition or removal of propositions or by the addition and deletion of formulas. The advantage of logical representation is that it is straight forward with rules for the creation and modification of knowledge. Inferences too can be made using procedures. For simple constructs, logical representation works without much flaws. The simplicity of the logical representation, like mathematical formulas, can be grasped easily. Moreover, the proposition is represented once but can be used in many formulas. The drawback of logical representation is that it is difficult to use it for large and complex sentences. Moreover, there are not hierarchical organisation of knowledge on logic based KBS.

\subsection{Semantic Networks}

Semantic networks use directed graph representation with concepts or nodes or vertices which are linked using edges or relationships. The knowledge in semantic network is
The challenge of most rule based and model based KBS is

organised hierarchically and the sub-nodes inherit properties from its super-nodes. Knowledge is added by inserting nodes and labelled relationships. Information is retrieved from the semantic network based KBS by getting the concept and the path connected to the concept. This allows a direct access to the knowledge in the knowledge base. Moreover, the graphical representation helps present the knowledge in a more readable format. The problem with semantic web is the lack formal semantic representation techniques. Syntactic methods have to be used to represent concepts and relationships. For the representation of complex sentences, the semantic networks will not be very helpful.

\subsection{Frames}

Frames are similar to semantic networks with the nodes of the semantic network being represented by units in frames. Each unit and its associated properties and units form frames which acts as a unit of knowledge. Similar to the semantic network, the units in frames inherit knowledge from within a frame or from other frames. The advantage of frames over semantic network is that frames are able to represent complex sentences by breaking them down to simpler sentence and linking the different frames of the simple sentences. Compound sentences be represented in a single frame. Frames can also be represented using relational databases.

There are other forms of knowledge representation like the procedural representation where the knowledge is represented if the form of procedures or production rules that have to be followed for knowledge acquisition or retrieval.

\section{RELATIONAL DATABASE AND GRAPH DATABASE \& RESULTS}

Relational databases and graph databases can both be used for knowledge base creation and utilisation. Traditionally the relational databases were used when the knowledge representation where logic based. With the popularity of the semantic networks rising, the graph databases began to become simultaneously popular. The frames can use both the relational and graph databases but tend to rely more on the robust relational databases.

\subsection{Relational Database}

Relational databases, introduced by Edgar Codd in 1970, began as a storage structure to help first order logic. It has found application mainly in relational algebra and relational calculus. The data object is stored in the form of a table in which the rows hold the records and the columns represents the fields. The rows and columns are related to the domain. The properties of an object can be held in different tables while the object itself becomes the primary key to identify its properties. The advantage of the relation database is that it can store huge amount of data and a search for an object and its properties is relatively faster. So, the information retrieval using relation database is quicker in huge datasets. [13]-[14]

$$
\begin{aligned}
& \text { Published By: } \\
& \text { Blue Eyes Intelligence Engineering } \\
& \text { \& Sciences Publication }
\end{aligned}
$$




\subsection{Graph Database}

The graph databases began in response to the huge amount of linked data available through the internet. The objects in a graph are nodes that are linked to each other by the vertices that gives a labelled relationship between the nodes. The properties of an object in the graph are all the connected nodes together with the connections or relationships. The popularity of the graph databases grew mainly because of its similarity with the real world approach. The ability of the graph databases to reliably link big data is an added advantage. There are many approaches to graph database representation all of which are classified as the NoSQL representation of data. [13]-[15]

\subsection{Hybrid Database}

Hybrid database is the more recent attempt to combine the relational and graph database approaches. The combination of both the approaches tries to overcome limitations of individual approaches and also allows the migration from one approach to the other. Certain hybrid approaches allow data to be stored in their original form without migrating from one form to the other. This enables building of applications irrespective of the form of database. The portability of such applications helps in a wider use of it. [16][17] The complexity of such hybrid approaches are much greater than its advantages and use.

\section{DISCUSSION AND CONCLUSION}

Knowledge representation has been specialised field of study and research from the time that artificial intelligence as permeated all domains of information and knowledge. Knowledge representation is part of knowledge engineering and as such is a vital for any knowledge based systems. With vast amount of information available on the internet, a system of knowledge acquisition and representation is important for the application of the information. Study and researches have been done and lot of literature on the topic of knowledge representation has been produced. The paper is a preliminary introduction to the topic of knowledge representation and its use in knowledge base system. It could be further developed with various advance made and more research can be done to find an efficient and reliable knowledge representation technique

\section{REFERENCES}

1. F. Roda and E. Musulin, An Ontology-based Framework to Support Multivariate Qualitative Data Analysis, vol. 33. Elsevier, 2014.

2. F. Schmalhofer, "Expert Systems in Cognitive Science," in International Encyclopedia of the Social \& Behavioral Sciences, Elsevier, 2001, pp. 5128-5135.

3. F. A. Cummins, "Rules for Actions and Constraints," in Building the Agile Enterprise, Elsevier, 2017, pp. 155-182.

4. S. Edelkamp and S. Schrödl, "Automated System Verification," in Heuristic Search, Elsevier, 2012, pp. 701-736.

5. A. Valente and J. A. P. J. Breuker, "Legal Knowledge Based Systems," in The Foundation for Legal Knowledge Systems, C. A. F. M. Grütters, J. A. P. J. Breuker, H. J. Herik, Van den, A. H. J. Schmidt, and C. N. J. De Vey Mestdagh, Eds. IOS Press, 1992, pp. 123-134.

6. M. Fedorova et al., Development of Computer Aided Modelling Templates for Model Re-use in Chemical and Biochemical Process and Product Design: Import and export of models, vol. 37, no. June. Elsevier, 2015.

7. P. Harmon, "AI-driven process change," in Business Process Change, Elsevier, 2019, pp. 417-439.
8. M. A. Tayal, M. M. Raghuwansh, and L. Malik, "Knowledge representation: Predicate logic implementation using sentence-type for natural languages," in 2013 International Conference on Circuits, Power and Computing Technologies (ICCPCT), 2013, pp. 1264 1269.

9. J. Mylopoulos and H. Levesque, "An Overview of Knowledge Representation,” 1983, pp. 143-157.

10. K. De Smedt, "Knowledge Representation Techniques in Artificial Intelligence: An Overview," in Human-Computer Interaction, Berlin, Heidelberg: Springer Berlin Heidelberg, 1988, pp. 207-222.

11. P. Tanwar, T. V. Prasad, and M. S. Aswal, "Comparative Study of Three Declarative Knowledge Representation Techniques," nternational J. Comput. Sci. Eng., vol. 2, no. 7, pp. 2274-2281, 2010.

12. M. R. Mohapatra and J. R. Mohanty, "An Overview of Knowledge Representation Techniques,” Int. J. Sci. Res. Comput. Sci. Eng. Inf. Technol., vol. 3, no. 5, pp. 490-495, 2018.

13. S. Medhi and H. Baruah, "Relational database and graph database: A comparative analysis," J. Process Manag. New Technol., vol. 5, no. 2 , pp. 1-9, 2017.

14. A. Martinez, R. Mora, D. Alvarado, G. López, and S. Quiros, "A Comparison between a Relational Database and a Graph Database in the context of a Personalized Cancer Treatment Application," in Proceedings of the Alberto Mendelzon International Workshop on Foundations of Data Management, 2016.

15. G. Jaiswal, "Comparative analysis of Relational and Graph databases," IOSR J. Eng., vol. 03, no. 08, pp. 25-27, Aug. 2013.

16. H. R. Vyawahare, P. P. Karde, and V. M. Thakare, "A Hybrid Database Approach Using Graph and Relational Database," in 2018 International Conference on Research in Intelligent and Computing in Engineering (RICE), 2018, pp. 1-4.

17. Y. Unal and H. Oguztuzun, "Migration of data from relational database to graph database," in Proceedings of the 8th International Conference on Information Systems and Technologies - ICIST '18, 2018, pp. 1-5. 